> Les protéines à domaine START représentent une famille de quinze membres chez les mammifères. Chez l'homme, sur la base d'homologies structurelles, cette famille est divisée en plusieurs sousgroupes comprenant principalement des transporteurs intracellulaires non vésiculaires de stérols et de lipides. À l'exception du groupe constitué par les thioestérases à domaine START, les différents groupes sont représentés chez les invertébrés. Le domaine START, toujours situé à l'extrémité carboxy-terminale des protéines, est un module d'environ 210 résidus qui lie les lipides, y compris les stérols. Le cholestérol, le 25-hydroxycholestérol, la phosphatidylcholine, la phosphatidyléthanolamine et les céramides sont les ligands identifiés pour respectivement STARDI/STARD3-6, STARD5, STARD2/STARD10, STARD10 et STARD11, les lipides ou stérols liés par les 7 autres protéines START sont inconnus. Le domaine START est considéré comme un domaine d'échange ou senseur de lipide ou de stérol. Il est constitué d'une poche amphiphile obturée par une hélice $\alpha$ faisant office de couvercle qui isole le ligand hydrophobe du milieu aqueux. Au sein du même sous-groupe, comme celui des transporteurs de stérols, les domaines START de différentes protéines ont des propriétés biochimiques similaires. Cependant, leur profil d'expression, leur régulation et leur localisation subcellulaire les distinguent et déterminent leurs différentes fonctions biologiques. Les protéines à domaines START interviennent dans des processus physiologiques distincts, tels que les échanges de lipides et de stérols entre compartiments intracellulaires, le métabolisme des lipides et la transduction du signal. La mutation ou la dérégulation de l'expression de certaines protéines à domaine START sont liées à divers processus pathologiques comprenant des troubles génétiques, autoimmuns et les cancers. <

\section{Les protéines à domaine START, des trafiquants intracellulaires de lipides}

Fabien Alpy, François Legueux, Laurent Bianchetti, Catherine Tomasetto

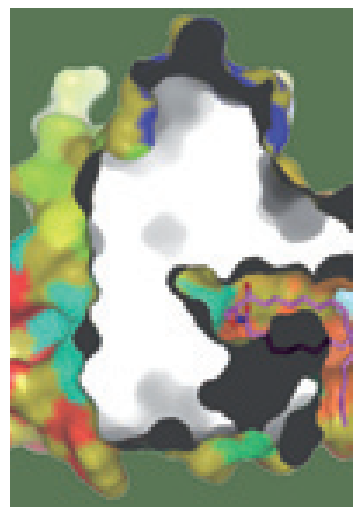

F. Alpy, F. Legueux, C. Tomasetto : Institut de Génétique et de Biologie Moléculaire et Cellulaire (IGBMC), Département de Biologie du Cancer, UPR 6520 CNRS/U964 Inserm/Université Louis Pasteur, BP10142, 67404 IIIkirch, CU de Strasbourg, France. L. Bianchetti : Plate-forme Bio-informatique de Strasbourg, Laboratoire

de Bio-informatique et Génomique Intégratives, Le terme général IGBMC, BP10142, 67404 IIIkirch, «lipide» englobe CU de Strasbourg, France. diverses molécules cat@igbmc.u-strasbg.fr caractérisées par une absence ou une faible solubilité dans l'eau. Les principaux lipides sont les acides gras, les phospholipides, les glycolipides et les stérols. Au même titre que les acides nucléiques et les protéines, les lipides sont des constituants essentiels des organismes supérieurs. À l'échelle de l'organisme, ils assurent de multiples fonctions: ils sont par exemple sources d'énergie et précurseurs de médiateurs humoraux. À l'échelle de la cellule, les lipides sont des composants structuraux des membranes et ils conditionnent la mise en place et le maintien des compartiments intracellulaires.

Le caractère peu soluble de ces molécules implique un mode de transport complexe, assuré au niveau de l'organisme principalement par des lipoprotéines plasmatiques et au niveau de la cellule par des échanges vésiculaires et non vésiculaires [1]. C'est dans le mode de transport non vésiculaire qu'interviennent les protéines à domaine START [ (steroidogenic acute regulatory protein) related lipid transfer] qui ont été nommées STARD (START domain containing) [2, 6]. Le domaine START est un module protéique d'environ 210 acides aminés liant les lipides, nommé par référence à la protéine steroidogenic acute regulatory protein (StAR/STARD1), 
un transporteur mitochondrial du cholestérol. Ce domaine est conservé au cours de l'évolution; il est capable de lier différents lipides dont les stérols, les phospholipides et les céramides.

\section{Diversité des protéines à domaine START}

\section{Six sous-familles de protéines}

à domaine START chez les mammifères

L'identification de la protéine steroidogenic acute regulatory protein (StAR/STARD1) une protéine essentielle à la stéroïdogenèse, et celle de la protéine Metastatic lymph node 64 (MLN64/STARD3) surexprimée dans les cancers du sein, ont révélé la présence d'un domaine conservé dans leur partie carboxy-terminale [3-5]. Cette région d'homologie conservée au cours de l'évolution et présente dans d'autres protéines a été nommée le domaine START (pour StAR-related lipid transfer) [2, 6]. Chez l'homme, un domaine START toujours situé en position carboxy-terminale est présent dans 15 protéines différentes [2]. Un alignement multiple des 15 domaines START humains permet de construire un arbre phylogénétique montrant une division de cet ensemble de protéines en six sous-familles (Figure 1). La nomenclature de ces

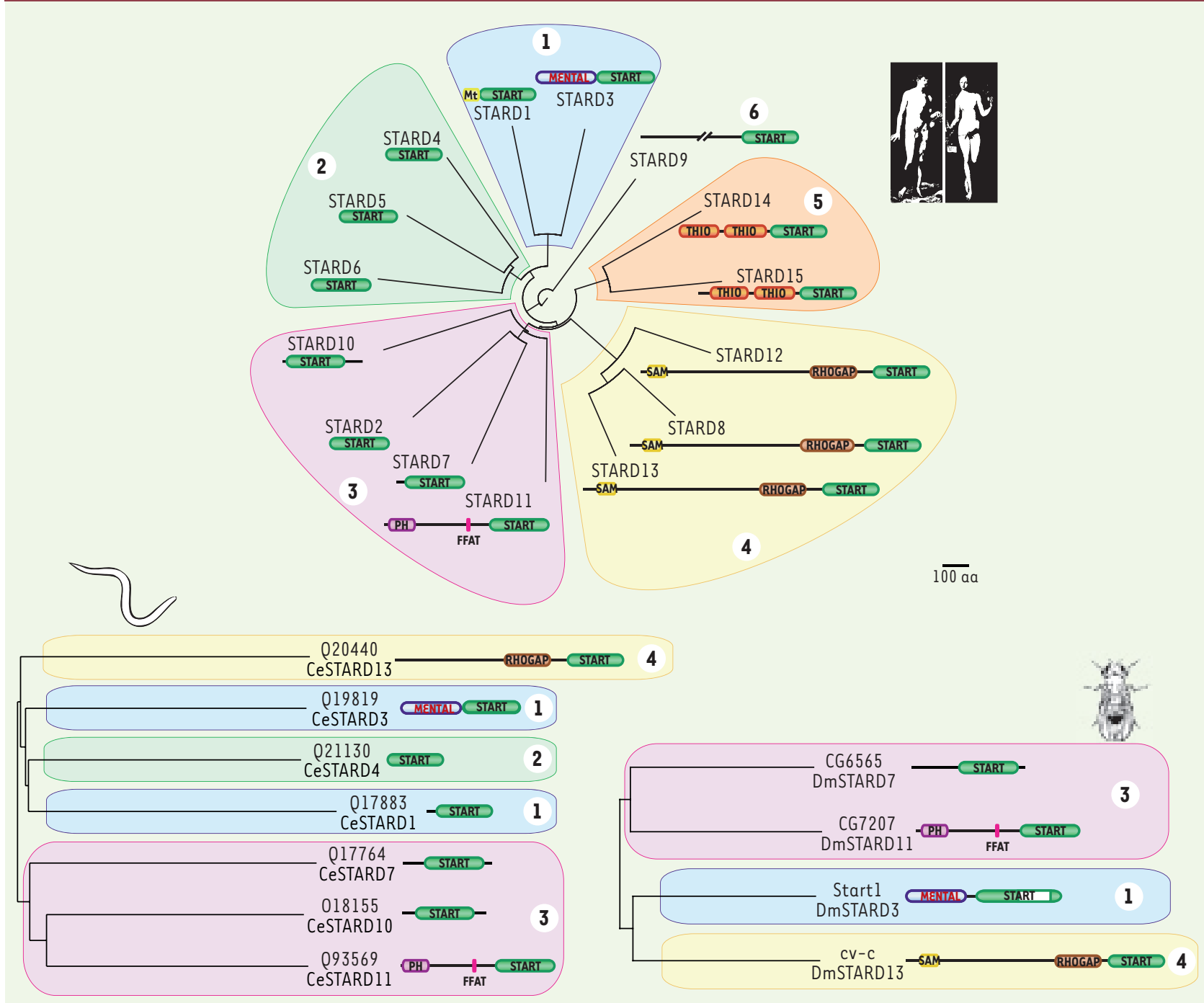

Figure 1. Arbre phylogénétique des protéines à domaine START chez l'homme (Homo sapiens), la mouche (Drosophila melanogaster) et le ver (Caenorhabditis elegans). L'alignement des séquences des domaines START des protéines humaines STARD permet de subdiviser cette famille en 6 sous-familles. Mt, signal d'adressage à la mitochondrie ; MENTAL, domaine MLN64 N-terminal ; PH, domaine d'homologie à la pleckstrine ; FFAT, motif de deux phénylalanines dans un contexte d'acides aminés acides responsable de l'adressage au réticulum endoplasmique; RHOGAP, domaine activateur de Rho-GTPases; SAM, motif alpha stérile; THIO, domaine acyl-CoA thioestérase. Pour la mouche et le ver, espèces pour lesquelles peu de données fonctionnelles existent, l'identification des protéines, basée sur l'homologie de séquence, a été faite par rapport à l'orthologue le plus proche chez l'homme. 


\begin{tabular}{|c|c|c|c|c|c|}
\hline & Nom & Autre noms $\mathbf{a}$ & Principaux acronymes $b$ & Spécificité de ligandc & Locusd \\
\hline \multirow{2}{*}{1} & STARDI & Steroidogenic Acute Regulatory protein & StAR & Chol & $8 p 11.2$ \\
\hline & STARD3 & Metastatic Lymph Node 64 & MLN64, CABl & Chol & $17 q 11-q 12$ \\
\hline & STARD4 & - & - & Chol & $5 q 22.1$ \\
\hline \multirow{3}{*}{2} & STARD5 & - & - & Chol, 25-OH Chol & $15 q 26$ \\
\hline & STARD6 & - & - & Chol & $18 q 21.2$ \\
\hline & I STARD2 & Phosphatidyl Choline Transfer protein & PCTP & $P C$ & $17 q 21-q 24$ \\
\hline \multirow{4}{*}{-3} & STARD7 & Gestational trophoblastic tumorl & GTII & $?$ & $2 q 11.2$ \\
\hline & STARD 10 & - & PTCP-like, SDCCAG28, CGI-52 & $P C, P E$ & $11 q 13$ \\
\hline & STARDII & Ceramide Transport protein & CERT, GPBP, COL4A3BP & Céramide & $5 q 13.3$ \\
\hline & STARD8 & Deleted in Liver Cancer-3 & DLC-3 & $?$ & Xq13.1 \\
\hline \multirow[t]{2}{*}{4} & STARD12 & Deleted in Liver Cancer -1 & DLC-1,Arhgap7, p122-RhoGAP & ? & $8 p 22$ \\
\hline & STARDI3 & Deleted in Liver Cancer -2 & DLC-2, SDCCAG13 & ? & $13 q 12-q 13$ \\
\hline \multirow{2}{*}{5} & STARDI4 & Brown fat inducible thioesterase & BFIT, THEA, ACOTIl & $?$ & $\operatorname{lp} 32.3$ \\
\hline & STARDI5 & Cytoplasmic acetyl-CoA hydrolase & $\mathrm{CACH}, \mathrm{ACOT} 12$ & $?$ & $5 q 14.1$ \\
\hline 6 & \{ STARD9 & - & - & $?$ & $15 q 15.1-q 15.2$ \\
\hline
\end{tabular}

Tableau I. Les protéines à domaine START chez l'homme : nomenclature, ligands et localisation chromosomique. Le code couleur correspond à celui de la Figure 1. a : autre noms complets les plus courants. b : Abbréviations communes : StAR : steroidogenic acute regulatory protein; PCTP : phosphatidyl choline tranfer protein ; MLN : metastatic lymph node ; CAB : coamplified with erbB2; GT : gestational trophoblastic tumor ; SDCCAG : Serologically defined colon cancer antigen; GPBP : goodpasture antigen binding protein; COL4A3BP : Collagen alpha 3 (IV) chain binding protein ; CERT : CERamide Transport ; DLC : deleted in liver cancer; ACOT : Acyl-CoA thioesterase ; CACH : Cytoplasmic acetyl-CoA hydrolase ; BFIT : Brown fat inducible thioesterase ; THEA : thioesterase adipose associated. b Ligands: Chol : Cholestérol ; 25-OH Chol : 25 Hydroxycholestérol ; PC : phosphatidyl choline; PE : phosphatidyl ethanolamine. $\mathrm{d}$ : localisation des gènes selon Unigene mapping position (NCBI).

protéines est assez hétéroclite, aussi avons nous adopté une nomenclature simplifiée, les différents noms des protéines START sont rassemblés dans le tableau I.

\section{Le groupe STARD1/StAR}

\section{de transporteurs intracellulaires de cholestérol}

Ce groupe comprend deux membres, STARDI (StAR), le membre fondateur de la famille, et STARD3 (MLN64). STARDI et STARD3 diffèrent dans leur partie aminoterminale, STARDI possédant un signal d'adressage à la mitochondrie alors que STARD3 possède un domaine d'ancrage membranaire, le domaine MENTAL (MLN64 amino-terminal) [3, 7]. STARDl est une protéine essentielle à la stéroïdogenèse [8]. Isolés, les domaines START de STARDI et de STARD3 ont des propriétés biochimiques identiques de liaison et de transfert du cholestérol $[4,9]$. Ils agissent de manière similaire sur la stéroïdogenèse en rendant le cholestérol initialement présent dans la membrane externe de la mitochondrie accessible au complexe P450scc présent sur la membrane interne [9]. En revanche les profils d'expression et les localisations subcellulaires des protéines STARDI et STARD3 les distinguent. En effet STARDI est une protéine mitochondriale principalement exprimée dans les gonades et les surrénales alors que STARD3 est ancrée à la membrane des endosomes tardifs et est exprimée de manière ubiquitaire $[3,10]$.

\section{Le groupe des autres transporteurs de stérols}

II comprend 3 protéines STARD4, STARD5 et STARD 6 qui sont formées uniquement du domaine START. L'identifi- cation du gène STARD4, sous exprimé par le foie de souris soumises à un régime riche en cholestérol, a permis de révéler ce groupe de trois gènes très homologues [11]. Les trois protéines codées par ces gènes lient le cholestérol et vraisemblablement les oxystérols. Les propriétés de liaison du cholestérol de STARD4/D5 et D6 sont sensiblement identiques à celles de STARD 1 et $D 3$. Cependant, seule STARD6 possède une activité similaire voire supérieure à celle de STARDI [9]. De manière intéressante STARD6 est presque exclusivement exprimée par les cellules germinales mâles, de ce fait elle ne remplace pas l'activité de STARD1 qui, elle, est restreinte aux cellules stéroïdogènes des gonades (cellules endocrines) [2, 12]. La fonction réelle de STARD6 reste incomprise mais elle est potentiellement impliquée dans le transfert du cholestérol vers la mitochondrie ou les autres membranes des cellules germinales mâles.

\section{Le groupe STARD2/PCTP, des transporteurs de lipides}

L'analyse phylogénétique associe STARD2/PCTP, STARD7, STARD10 et STARD11/CERT/GPBP. Ce groupe est plus hétérogène que les autres par l'organisation structurale des protéines et leur spécificité de ligand. Si STARD2, STARD7 et STARD10 sont des protéines composées uniquement d'un domaine START, STARDIl possède deux autres domaines fonctionnels d'interaction avec les membranes, un domaine PH (pleckstrin homology) et un motif FFAT (two phenylalanine in an acidic tract) ainsi qu'un domaine potentiel coiled-coil et deux motifs riches en sérines [13]. STARD2 et D10 lient toutes deux la phosphatidylcholine $(P C)$ et STARD10 lie également la phosphatidyléthanolamine (PE) [14, 15]. Il est vraisemblable que STARD7 lie les phospholipides. En revanche, STARD11 lie les céramides [16]. Ces quatre protéines ont une expression ubiquitaire, suggérant qu'elles remplissent un rôle général dans les transports de lipides. 
STARD2 est une protéine cytoplasmique qui promeut l'échange rapide de la phosphatidylcholine (PC) supposément de son lieu de synthèse vers la face interne de la membrane plasmique et/ou la membrane externe de la mitochondrie. STARD2 serait responsable du remplissage de la membrane plasmique par de la PC en réponse à l'efflux de phospholipides vers les lipoprotéines plasmatiques de type HDL (high density lipoproteins) au cours du transport des HDL entre les tissus $[17,18]$. In vitro, STARD10 permet l'échange membranaire de PC et PE [14]. En revanche peu de choses sont connues concernant STARD7 qui est exprimée par les cellules du trophoblaste suggérant un rôle dans le transport des lipides dans le placenta [19]. De manière surprenante, les souris déficientes pour STARD2 ont un phénotype quasiment normal [20]. Soumises à un régime riche en graisses et en cholestérol, les animaux STARD2 ${ }^{-/-}$ont des difficultés à éliminer les excès de PC, d'acides biliaires et de cholestérol par la bile [21], suggérant l'implication physiologique de cette protéine dans le métabolisme des phospholipides. Rappelons que STARD2, STARD7 et STARD10 sont co-exprimées dans le foie, et ces deux dernières pourraient compenser l'absence de STARD2 chez les souris STARD2 ${ }^{-/-}$[20].

STARD11 permet le transport non vésiculaire des céramides [16] de leur lieu de synthèse le réticulum endoplasmique (RE) vers l'appareil de Golgi où ils sont convertis en sphingolipides. STARDll peut transférer efficacement plusieurs espèces de céramides possédant de longues chaînes d'acide gras (C14-C20), du C16-dihydrocéramide et C16-phytocéramide [22]. La fonction de STARD1l implique la coopération de plusieurs domaines protéiques comme les domaines PH et FFAT qui sont instrumentaux dans le positionnement de la protéine. Seul le domaine START est impliqué dans le transfert des céramides d'une membrane donneuse à une membrane accepteuse [16].

\section{Le groupe des protéines Rho-GAP-START}

Ce groupe comprend 3 protéines: STARD8 (DLC-3), STARD12 (DLC-1) et STARD13 (DLC-2) dont les isoformes les plus longues possèdent toutes un domaine SAM (sterile alpha motif) du coté amino-terminal, un domaine Rho-GAP (Rho-GTP activating protein) dans la partie centrale et un domaine START du coté carboxy-terminal. Le domaine SAM est l'un des motifs protéiques les plus fréquents, c'est un domaine d'homo- ou d'hétéromérisation. Les domaines Rho-GAP sont impliqués dans la régulation des GTPases. Les GTPases Rho appartiennent à une famille très étendue, apparentée au proto-oncogène Ras qui comprend 3 sous-familles Ras, Rho et Rab [23]. La spécificité de ligand des domaines START de STARD8/12 ou D13 n'est pas connue. Ces protéines ont pourtant un rôle important comme en atteste le phénotype embryonnaire létal engendré par la délétion du gène STARD12 chez la souris [24]. Les trois protéines STARD8, D12-13 ont une forte similitude suggérant qu'elles exercent des fonctions proches et que leurs gènes dérivent du même ancêtre. STARD12 et D13 agissent sur le cytosquelette d'actine et réduisent la motilité cellulaire. Parmi les différents domaines structuraux composant ces protéines, c'est l'action du domaine Rho-GAP qui prédomine en inhibant l'activation des protéines Rho ou en interférant avec la balance GDP-GTP. Le domaine START pourrait avoir un rôle régulateur du domaine Rho-GAP. Ainsi, le domaine START pourrait activer le domaine Rho-GAP de ces protéines en fonction de sa liaison ou non à un ligand, jouant ainsi le rôle d'un senseur de lipide. Même si les trois gènes, STARD8, D12 et D13, sont exprimés par plusieurs tissus, leurs profils d'expression ne se superposent pas, offrant une explication à l'absence de compensation du phénotype embryonnaire létal des souris STARD12 $2^{-/-}$.

\section{Le groupe des protéines START \\ à domaine thioestérase}

Ce groupe est constitué de deux protéines STARD14 (acyl-CoA thioestérase 11/BFIT/THEA) et STARD15 (acyl-CoA thioestérase 12/CACH), proches (50\% d'identité et $68 \%$ de similitude), mal caractérisées, formées du coté amino-terminal de deux domaines enzymatiques (THIO) de type sérine-estérase similaires aux acétyl-CoA thioestérases des procaryotes, et du coté carboxy-terminal d'un domaine START. Leurs activités catalytiques sont peu connues: STARD14 hydrolyserait préférentiellement des substrats de type C12-16-CoA alors que STARD 15 hydrolyse plutôt l'acétyl-CoA $[2,6]$. Le ligand de leur domaine START est inconnu. L'expression de STARD14 est ubiquitaire, celle de STARD15 plus restreinte ce qui suggère que ces protéines ne sont pas redondantes. Compte tenu de leur activité enzymatique et de la présence du domaine START, il est vraisemblable que ces protéines aient un rôle de senseur permettant de réguler le métabolisme du cholestérol et des acides gras.

\section{L'énigme STARD9}

Rien n'est connu au sujet de STARD9, mis à part la présence d'un domaine START du coté carboxy-terminal et d'un cadre ouvert de lecture qui engendrerait une protéine de grande taille (Figure I).

\section{Le domaine START, un domaine conservé chez les pluricellulaires}

Ce domaine protéique est conservé au cours de l'évolution chez les plantes et les animaux mais il est absent chez les levures et les Archaebactéries [25]. II est cependant présent chez certains protistes et certaines Eubactéries. Chez les plantes, le domaine START est plus fréquent que chez les animaux, en particulier dans des facteurs de transcription à homéodomaine. La mouche a quatre gènes codant des protéines à domaine START et le ver en a six, répartis chez les deux espèces entre les sous-familles 1,3 et 4 (Figure 1). L'analyse phylogénétique montre que les protéines à domaine START se distinguent plus par leur spécificité vis-à-vis du 
ligand que par leur spécificité d'espèce (Figure 1). Cela suggère que la spécification du domaine vis-à-vis du ligand est antérieure à la séparation des espèces; il s'agit probablement d'un événement très ancien. Chez la mouche le transport des stérols est assuré par Startl ou DmSTARD3. Curieusement, chez le ver à soie, par un jeu d'épissage alternatif, ce gène, code pour deux isoformes exprimées différemment, l'une ressemblant à STARD3 est formée d'un domaine MENTAL et d'un domaine START, l'autre, ressemblant à STARDl, est limitée au domaine START. Ce dernier est impliqué dans le transport des caroténoïdes, en particulier dans la glande séricigène où il permet la coloration de la soie. Ce mécanisme d'épissage alternatif, s'il est conservé, permet la synthèse de plusieurs protéines START à partir d'un même gène. Les protéines START du ver sont très mal connues; de manière intéressante, STARD4 a été identifiée par un criblage d'ARN interférents à grande échelle comme régulant la masse graisseuse.

La forte conservation du domaine START au cours de l'évolution et sa diversification indiquent qu'il joue un rôle important dans le métabolisme et le trafic des lipides.

\section{Bases structurales de l'interaction protéine-lipide}

La structure cristallographique des domaines START des protéines STARD2, STARD3, STARD4 et STARD1l a été déterminée et montre le même type de spécificité structurale $[2,26]$. Elles adoptent un repliement de type helix-grip dans lequel un feuillet $\beta$ antiparallèle central est ceinturé par les hélices $\alpha$ amino- et carboxy-terminales ( $\alpha$ l et $\alpha 4$ ), cette dernière étant étroitement refermée sur les 9 brins du feuillet $\beta$ courbe (Figure 2). Deux boucles $\Omega$ sont insérées entre les brins $\beta 5$ et $\beta 6(\Omega 1)$ et les brins $\beta 7$ et $\beta 8(\Omega 2)$. L'organisation tridimensionnelle du domaine forme une cavité interne amphiphile. Les domaines START de STARD2 et de STARD1l ont été co-cristallisés avec le ligand et montrent une molécule de ligand à l'intérieur de la poche [26]. La cavité possède une entrée à l'interface du feuillet $\beta$ et de l'hélice $\alpha 4$, et la partie hydrophobe du lipide est en contact avec la partie hydrophobe de la cavité du domaine. Au fond de la cavité, la partie hydrophile du lipide établit des contacts avec des résidus polaires ou chargés de la protéine. L'entrée d'une molécule de ligand dans le domaine START ne peut se faire que si des réarrangements structuraux majeurs ont lieu, qui impliquent probablement les hélices $\alpha 3$ et $\alpha 4$ ainsi que la boucle $\Omega 1$.

La détermination de la structure tridimensionnelle de deux protéines de plante liant des lipides, Bet vl and Pru avl, a montré une organisation très similaire de leur structure bien qu'elles ne partagent pas de similitude de séquence avec les protéines à domaine START [2]. Ce type de repliement semble donc être commun à certaines protéines liant les lipides; il permet de créer une cavité amphiphile qui protège et oriente le ligand de manière à favoriser les échanges avec les membranes.

\section{Mécanisme moléculaire d'échange de lipide}

\section{Profil d'expression et localisation subcellulaire}

Au sein du même sous-groupe, les domaines START de différentes protéines ont des propriétés biochimiques similaires, cependant leur profil d'expression, leur régulation et leur localisation subcellulaire les distinguent. Ces composantes majeures vont déterminer leurs fonctions biologiques, elles ne sont hélas connues que pour peu d'entre elles. Deux catégories de protéines START se différencient selon qu'elles s'expriment de façon ubiquitaire ou avec une spécificité tissulaire. Ces dernières incluent les transporteurs de cholestérol comme STARDl et STARD6. STARDl est exprimée dans les organes stéroïdogéniques comme la glande surrénale, les gonades et le cerveau, où son expression est étroitement contrôlée au niveau transcriptionnel [27]. L'expression de STARD6 est restreinte aux cellules germinales mâles et au cerveau $[12,28]$. Ainsi, même dans les gonades mâles, l'expression des deux protéines ne se superpose pas, suggérant que la finalité du transport du cholestérol est différente même si le mécanisme moléculaire est conservé. La protéine STARD15, un membre de la famille

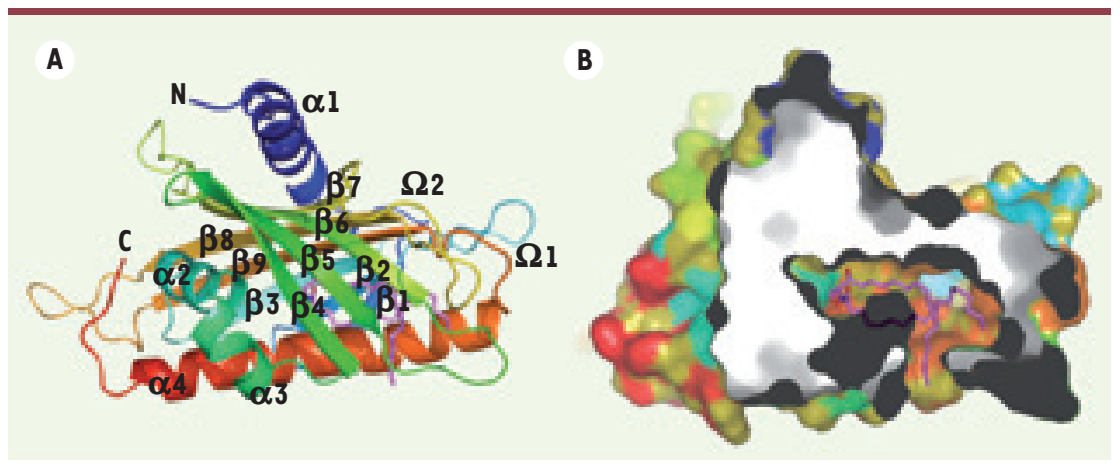

Figure 2. La structure du domaine START forme une cavité amphiphile dans laquelle se loge une molécule de lipide. A. Représentation en ruban de la structure du domaine START de la protéine STARDll complexé avec une molécule de céramide C18 (PDB ID : $2 e 3 q$ ) ; les hélices $\alpha(1-4)$, les feuillets $\beta$ (19 ) et les boucles $\Omega(1,2)$ sont numérotés du $\mathrm{N}$ au C-terminal. B. Visualisation de la molécule de céramide au sein du domaine START de STARDIl. Une coupe virtuelle de la surface du domaine START montre la molécule de céramide logée au sein du domaine protéique. Les atomes de $\mathrm{C}, \mathrm{N}$ et $\mathrm{O}$ de la molécule de céramide sont respectivement en violet, bleu et rouge. 


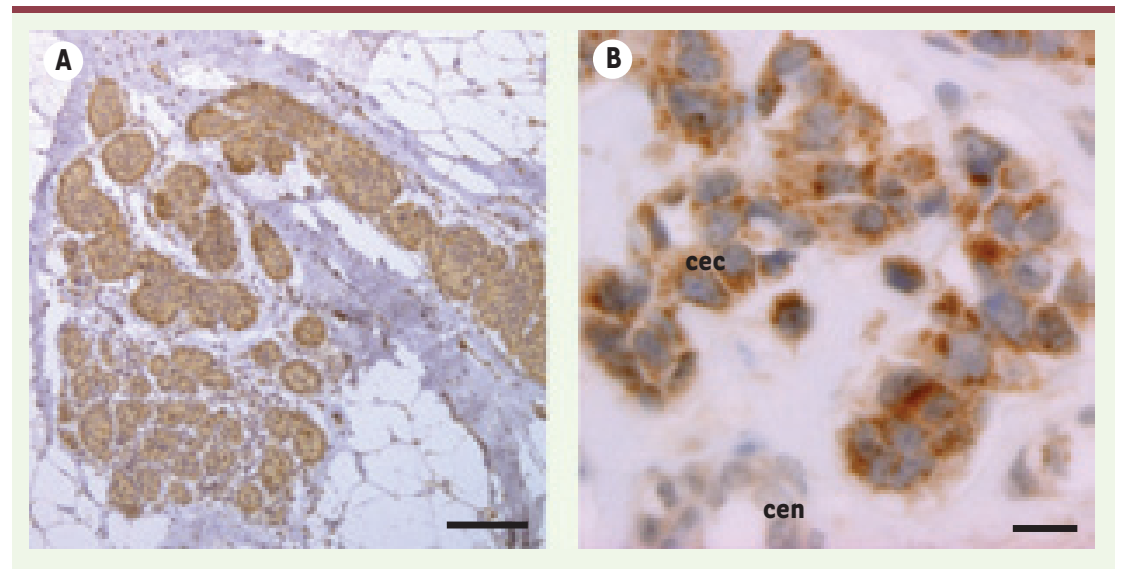

Figure 3. STARD3 est surexprimée dans les cancers mammaires. La protéine STARD3 (brun) est détectée par immunohistochimie sur des coupes de cancer canalaire invasif. À fort grossissement (droite), la protéine est sur-produite spécifiquement par les cellules épithéliales cancéreuses (cec) alors que les cellules épithéliales normales (cen) l'expriment plus faiblement à un niveau basal. Échelles : $200 \mu \mathrm{m}$ et $20 \mu \mathrm{m}$.

des protéines START à domaines thioestérase, est également exprimé préférentiellement dans le foie. Les autres protéines START, même si elles ne sont pas totalement ubiquitaires, ont des profils d'expression très larges. Par exemple le transporteur de cholestérol, STARD3, est exprimé par tous les tissus - ce qui est corroboré par la présence dans le promoteur de STARD3 de séquences consensus typiques d'un promoteur ubiquitaire [29] - mais certains tissus comme le placenta l'expriment de façon plus importante [4]. Certains organes importants pour le métabolisme des lipides comme le foie, expriment la plupart des protéines START suggérant qu'il existe une redondance et/ou une coopération entre ces protéines dans cet organe.

L'autre clé de la fonction de ces protéines tient à leur localisation subcellulaire. Les protéines START d'un même sous-groupe occupent des territoires cellulaires différents (Figure 4). Par exemple, la protéine STARDI est une protéine mitochondriale alors que STARD3 est localisée à la membrane des endosomes tardifs (Figure 4) [3, 10]. La position de STARD3 à la membrane des endosomes suggère qu'elle intervient dans la voie de recapture du cholestérol par les lipoprotéines plasmatiques [10]. Les protéines START-Rho-GAP sont des composants des complexes d'adhérence focale, structures qui lient le cytosquelette d'actine et la matrice extracellulaire [30]. En accord avec cette localisation, les cellules dépourvues de STARD12 ont des anomalies du cytosquelette et le domaine Rho-GAP intervient dans ce phénomène [24]. STARD12 est aussi localisée dans les cavéoles, des micro-domaines invaginés de la membrane plasmique riches en cholestérol et caractérisés par la présence de la protéine cavéoline [31]. Quelques protéines START sont cytosoliques, comme STARD2, STARD4, STARD5. Cependant, leur localisation peut être modulée selon différents types et/ou conditions cellulaires. Par exemple, dans une lignée cellulaire de macrophages hépatiques, STARD5, par ailleurs cytosolique, se localise au niveau de l'appareil de Golgi [32]. Quelques protéines START ont été localisées dans le noyau, c'est le cas de STARD6 pour les spermatocytes et le tissu nerveux $[12,28]$. Finalement c'est le transporteur des céramides STARD1l qui illustre le mieux la relation entre localisation et fonction des protéines START. STARDll possède deux motifs d'adressages ciblant des membranes respectivement riches et pauvres en céramides (Figure 4) et permet ainsi d'assurer le transport non vésiculaire de ce lipide [13].
Les protéines à domaine START sont probablement plutôt des transporteurs, dont la localisation est vraisemblablement dynamique entre plusieurs compartiments. La majorité des données actuelles reflète simplement la localisation à l'équilibre de protéines START surexprimées dans un modèle cellulaire. La localisation en temps réel des protéines endogènes dans un contexte physiologique nous permettra de mieux comprendre leurs rôles.

\section{Les modifications post-traductionnelles modulent l'activité des protéines START}

Le fonctionnement des protéines à domaine START peut être régulé par des modifications post-traductionnelles. Ainsi le domaine START de STARDl est phosphorylé sur la sérine 195 par la protéine kinase A ce qui augmente son activité de transport de cholestérol [2]. La protéine STARDll possède elle aussi des sites de phosphorylation dans un motif protéique contenant plusieurs sérines (motif de répétition de sérines) situé entre son domaine $\mathrm{PH}$ et son motif FFAT. La phosphorylation des sérines de ce motif par la protéine kinase PKD diminue l'activité de STARD1l, alors que leur déphosphorylation par la protéine phosphatase 2 C (PP2C) augmente l'association de STARD 11 avec le réticulum endoplasmique et le Golgi ainsi que le transport des céramides [33, 34].

\section{Changement conformationnel et rôle de l'hélice $\alpha 4$} Une autre fonction récurrente des protéines START outre le transport des lipides - est d'échanger ces lipides entre les membranes sans faire appel à des vésicules. La dynamique de ces échanges de lipides est mal comprise. Cependant, un modèle potentiel de fonctionnement de ces protéines peut être déduit des études effectuées sur les protéines STARDl et STARDIl. STARDl a un signal d'adressage mitochondrial à son extrémité amino-terminale qui est retiré lors de son import dans la matrice mitochondriale où la protéine 


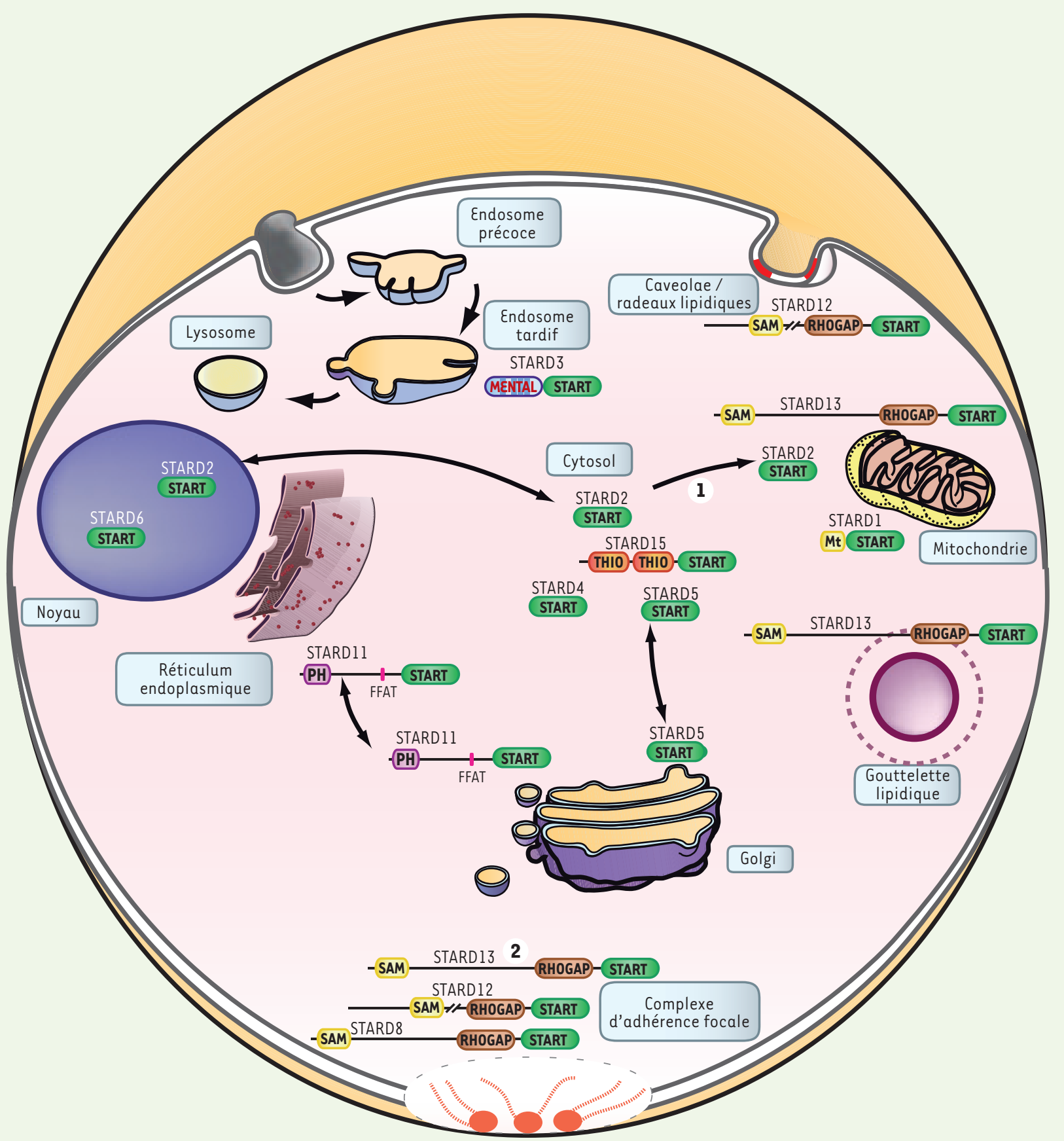

Figure 4. Distributions cellulaires connues des protéines à domaine START. Représentation schématique des différentes protéines à domaine START au sein d'une même cellule. La structure des protéines reprend le code adopté dans la Figure 1. STARD1 est une protéine mitochondriale. STARD2 est nucléo-cytoplasmique et s'accumule dans les mitochondries après traitement au clofibrate (1) dans des cellules endothéliales. STARD3 est ancrée dans la membrane périphérique des endosomes tardifs. STARD5 est un transporteur cytosolique mais une association avec le Golgi a été mise en évidence dans des macrophages. STARD 6 est nucléaire dans les cellules germinales mâles et dans les neurones. STARD8 est située au niveau des complexes d'adhérence focale. STARD11 effectue le trafic non vésiculaire des céramides du réticulum endoplasmique au Golgi. STARD12, comme STARD8 est localisé dans les complexes d’adhérence focale ainsi que dans des régions membranaires enrichies en cholestérol qui sédimentent dans les mêmes fractions que les cavéoles. L'interaction de STARD13 avec la tensine 1 suggère que, comme STARD12, elle est un composant des complexes d'adhérence (2). STARD13 a également été observée dans les mitochondries et dans une région proche des gouttelettes lipidiques. STARD15, de par, sa structure sans domaine d'adressage, est prédite cytosolique. Les localisations de STARD4, 7, 9, 10 et 14 sont toujours incertaines. 


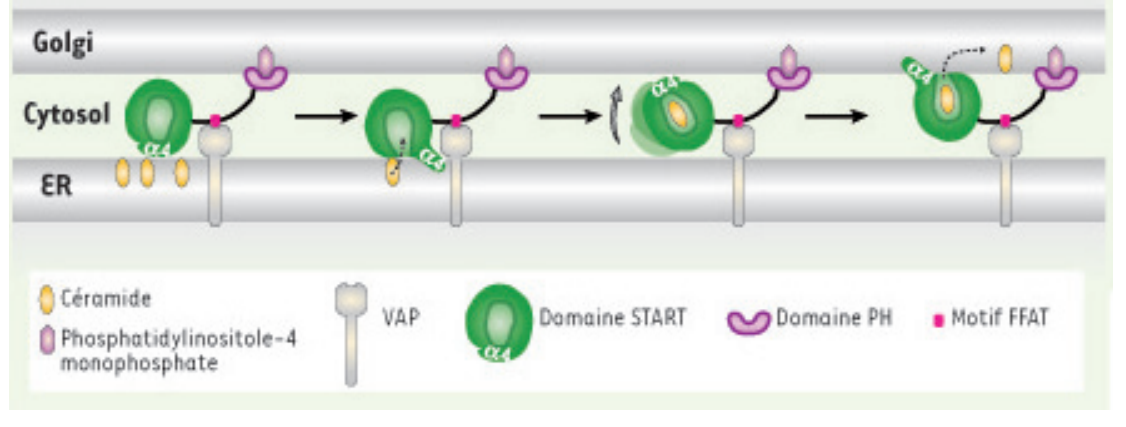

Figure 5. Modèle d'action de la protéine STARD11. La protéine STARDll grâce à son domaine PH interagit avec le phosphatidylinositole-4 monophosphate de l'appareil de Golgi et grâce à son motif FFAT avec la protéine VAP du RE. Par un mécanisme de flip-flop, la protéine STARD 11 pourrait extraire les céramides du RE et les transférer au Golgi.

STARDl est finalement dégradée [2]. De façon surprenante, une protéine STARDI dépourvue d'adressage mitochondrial car ne possédant pas ses 62 premiers acides aminés, garde une activité stéroïdogène in vitro. De plus, la protéine StAR est inactive lorsqu'elle est localisée de façon forcée dans la matrice de la mitochondrie ou dans l'espace inter-membranaire alors qu'elle garde son activité lorsque sa localisation est forcée à la membrane externe mitochondriale [35]. Des études biophysiques ont montré que la protéine STARDl adopte une conformation de globule fondu à faible $\mathrm{pH}$ et en association avec les membranes, un état structural dans lequel les structures tertiaires de la protéine sont dynamiques alors que les structures secondaires sont similaires à celles de la protéine native. Pour STARDl, cet état de globule fondu est associé à la libération du cholestérol. Finalement, d'autres études ont montré que I'hélice $\alpha 4$ du domaine START (Figure 2) de STARDl peut se lier aux membranes de façon $\mathrm{pH}$ dépendante. Ensemble, ces données sont en faveur d'un modèle selon lequel STARDl agit au niveau de la membrane externe de la mitochondrie. L'interaction entre l'hélice $\alpha 4$ du domaine START et les têtes protonées des phospholipides de la membrane externe mitochondriale change la conformation de la protéine et ouvre ainsi le couvercle formé par l'hélice $\alpha 4$, donnant accès à la cavité amphiphile du domaine START. En accord avec un rôle crucial de la dynamique de I'hélice $\alpha 4$, des mutants de STARDl possédant des ponts di-sulfures bloquant la mobilité de l'hélice $\alpha 4$ n'ont plus la capacité de transporter le cholestérol, alors que la réduction de ces ponts di-sulfures restaure le transport [36]. L'import de STARDI dans la mitochondrie ne serait donc que secondaire à son action et terminerait probablement la stéroïdogenèse. Ce modèle de fonctionnement peut être extrapolé à celui d'autres protéines START. Ainsi, la région carboxyterminale de STARD2 incluse dans l'hélice $\alpha 4$ a un rôle dans la liaison aux membranes et facilite l'extraction des PC.

Pour les protéines composées uniquement d'un domaine START, I'hélice $\alpha 4$, en interagissant avec une membrane, permet l'ouverture de la cavité de liaison au lipide du domaine. Après la prise en charge du lipide, la cavité est refermée et la protéine peut échanger son lipide avec une autre membrane. Le mécanisme d'adressage aux différentes membranes des protéines constituées uniquement du domaine START n'est pas clair. Il est beaucoup mieux compris pour les protéines possédant des sites d'adressage intrinsèques spécifiques d'organites tels que STARD3 et STARD1l. Ainsi, le domaine PH de ce dernier adresse la protéine au Golgi en interagissant avec les phosphatidylinositoles-4 monophosphate et un motif FFAT qui se lie à une protéine résidant dans le RE appelée VAP (vesicle-associated membrane protein-associated protein) (Figure 5). C'est le domaine START de la protéine qui est responsable de l'échange spécifique des céramides d'une membrane donneuse vers une membrane accepteuse. Ainsi, le domaine PH et le motif FFAT de STARD1l restreignent spatialement l'activité de transfert des céramides du domaine START. De façon similaire, dans les cellules transfectées, les tubules d'endosomes tardifs contenant STARD3 sont parallèles aux mitochondries positives pour STARDl et les contactent transitoirement [2]. En effet, le domaine MENTAL de STARD3 ancre la protéine à la périphérie des endosomes tardifs (Figure 1) et très probablement conditionne le transfert du cholestérol par cette protéine. On peut spéculer que des protéines possédant des motifs d'adressage comme STARD1, STARD3, STARD1l sont localisées à des sites de contact membranaires en interagissant avec des protéines ou des lipides spécifiques, et que le domaine START agit au niveau de ces sites de contact. Des sites de contact membranaires entre différents organites ont été identifiés en microscopie. Dans ce scénario, STARD1 l, grâce à son domaine PH et à son motif FFAT, pourrait rapprocher le Golgi du RE et ainsi extraire et délivrer les céramides par un mécanisme de flip-flop (Figure 5).

Le recrutement des protéines START au niveau de sites de contact membranaires pourrait réconcilier des observations contradictoires concernant leur fonction. Ainsi, par exemple, la protéine STARDI lie une molécule de cholestérol mais il a été montré qu'elle peut transporter 400 molécules de cholestérol/STARD1/min [2]. Si les sites donneurs et accepteurs sont rapprochés, une protéine START pourrait établir des échanges rapides et efficaces de ce ligand. Jusqu'à présent, seules des membranes ont été identifiées comme sites d'échange de lipides cependant, les protéines START pourraient échanger des lipides avec d'autres protéines. 


\section{Physiopathologies impliquant des protéines à domaine START}

Plusieurs pathologies sont associées à l'une ou l'autre des protéines START, la mieux connue est I'hyperplasie congénitale lipoïde des surrénales (lipoïd $C A H$ ), une maladie génétique dans laquelle de nombreuses mutations du gène STARDl ont été identifiées [37]. Cette maladie se manifeste par l'accumulation pathologique de cholestérol sous forme de gouttelettes lipidiques dans le cytoplasme des cellules endocrines des gonades et des glandes surrénales ainsi que par l'absence de synthèse d'hormones stéroïdes. Ce phénotype cellulaire évoque un défaut de transport et traduit l'incapacité des protéines STARDl mutantes à transporter le cholestérol vers la mitochondrie pour permettre sa conversion en prégnénolone, le précurseur des hormones stéroïdes. L'inactivation du gène STARDl chez la souris phénocopie l'hyperplasie congénitale lipoïde des surrénales [37]. La deuxième pathologie incriminant les protéines START est le cancer. En effet, l'expression de plusieurs protéines à domaine START est modifiée dans les cancers (Tableau II). Ainsi, les cancers du sein surexprimant le gène erbB2/HER2, codant pour un membre de la famille du récepteur à l'£GF (epidermal growth factor), surexpriment STARD3 (Figure 3) [5] et STARD10 [38]. Les gènes STARD3 et ERBB2 sont tous deux localisés sur le chromosome 17 en q12-q21, une région chromosomique fréquemment amplifiée dans les cancers du sein. La proximité des deux gènes et la présence d'éléments régulateurs identiques dans leur promoteur expliquent leur coexpression et suggèrent leur coopération lors de la transformation cancéreuse [29]. La surexpression de STARD10 dans les cancers du sein est assez large et ne se limite pas aux cas positifs pour ERBB2. Cependant un lien fonctionnel existe entre STARD10 et le récepteur de l'EGF (EGFR/ $\varepsilon r b B 1$ ), sous forme d'une coopération STARD10 et $\varepsilon$ rbBl dans les processus de transformation tumorale [38].

Certains gènes codant des protéines à domaine START sont quant à eux inactivés dans les cancers. Le gène STARD12 nommé à l'origine DLC-1 (deleted in liver cancer 1 ), est un suppresseur de tumeur localisé sur le chromosome 8 p21-22, une région sujette à des pertes d'hétérozygotie dans les cancers. Ce gène est perdu dans certaines tumeurs primaires $d u$ foie et du sein [40] et il est sous-exprimé dans des lignées cellulaires issues de tumeurs du foie, de sein, de côlon et de prostate [41, 42]. Les deux autres membres de la sous-famille des protéines START-Rho-GAP, STARD13 et STARD8 [39] sont eux aussi deux potentiels suppresseurs de tumeur. STARD13 est localisée en $13 q 12.3$, une région chromosomique présentant de fréquentes pertes alléliques dans les carcinomes hépatocellulaires et dans d'autres cancers [43]. II y a également une perte d'expression de STARD8 dans certains cancers. La forte conservation des ces trois gènes suggère qu'ils soient impliqués dans des fonctions similaires et celui dont la fonction est la mieux connue est STARD12.

L'expression forcée de STARD12 dans des lignées cellulaires de carcinomes de foie, de poumon et de sein diminue leur capacité de croissance, de former des colonies et des tumeurs chez des souris nude [41, 42, 44-46]. Des fibroblastes embryonnaires de souris inactivées pour le gène STARD12 montrent des altérations de l'organisation des filaments d'actine et des adhérences focales, ce qui prouve le rôle essentiel de cette protéine dans l'organisation du cytosquelette [24]. Ainsi, ce faisceau d'éléments indique que la perte de STARD12 et/ou des deux autres membres de ce sous-groupe pourrait contribuer aux changements d'organisation du cytosquelette fréquemment observés dans les cellules cancéreuses.

Enfin, la protéine STARDll a été impliquée dans différentes pathologies, un syndrome auto-immun d'une part et la résistance médicamenteuse d'autre part. STARD1l, le transporteur des céramides, fut historiquement nommé Goodpasture binding protein (GPBP) ou encore collagen alpha3(IV) chain binding protein (COL4A3BP). Ces deux derniers noms attestent de son implication dans le syndrome de Goodpasture [47].

\begin{tabular}{|c|c|c|c|}
\hline Nom & Autre noma & Impliqué dansb & Particularitésc \\
\hline STARDl & StAR & Hyperplasie congénitale lipoïde des surrénales ${ }^{1}$ & $\left\{\begin{array}{c}\text { Déficit en hormones stéroïdes et accumulation } \\
\text { de goutelettes lipidiques dans les organes stéroïdogènes }\end{array}\right.$ \\
\hline STARD3 & MLN64 & $\nearrow$ Cancer $^{2}$ & Amplification et surexpression dans les cancers du sein \\
\hline STARD7 & GTI & $\nearrow$ Cancer $^{3}$ & Surexpression lignées cancéreuses du trophoblaste \\
\hline STARD10 & PTCP-like & $\nearrow$ Cancer $^{4}$ & Surexpression dans les cancers du sein \\
\hline STARDII & $\begin{array}{l}\text { CERT, GPBP } \\
\text { COL4A3BP }\end{array}$ & $\begin{array}{l}\text { Syndrome de Goopasture }{ }^{5} \\
\text { Cancer }^{6}\end{array}$ & $\begin{array}{l}\text { Maladie autoimmune contre le domaine } \mathrm{NCl} \text { de la chaine a3 du collagène IV } \\
\text { Surexpression associée à la résistance médicamenteuse face aux agents anticancéreux }\end{array}$ \\
\hline STARD8 & DLC3 & $\searrow$ Cancer $^{7}$ & Sous-expression dans les cancers \\
\hline STARD12 & $\mathrm{DLCl}$ & $\searrow$ Cancer $^{8}$ & Gène perdu dans les tumeurs du foie et sous-expression dans les cancers \\
\hline STARD13 & DLC2 & $\searrow$ Cancer $^{9}$ & Sous-expression dans les cancers \\
\hline
\end{tabular}

Tableau II. Implications des protéines à domaine START dans certaines pathologies. Le code couleur correspond à la Figure 1. a : Abbréviations communes StAR : steroidogenic acute regulatory protein; MLN : metastatic lymph node; PCTP : phosphatidyl choline tranfer protein-like; GTT: gestational trophoblastic tumor; CERT : CERamide Transport protein ; GPBP : goodpasture antigen binding protein; COL4A3BP : Collagen alpha 3(IV) chain binding protein DLC : deleted in liver cancer. $b$ : Selon la littérature, $\lambda$ ou $\searrow$ signifie sur-exprimé ou sous-exprimé dans les cancers par rapport au tissu normal correspondant. $1:$ [37] ; $2:[5] ; 3:[19] ; 4:[14] ; 5:[47] ; 6:[50] ; 7:$ [39] ; $8:$ [41] ; 9 : [43]. c : Descriptions succinctes des altérations rapportées dans la littérature. 
Ce syndrome est caractérisé par la production d'autoanticorps dirigés contre le domaine $\mathrm{NCl}$ de la chaîne $\alpha 3$ du collagène de type IV, un constituant spécifique des membranes basales glomérulaires et alvéolaires [48]. Le lien entre STARDll et la pathologie n'est pas clair cependant plusieurs éléments l'incriminent. STARDll se lie au domaine $\alpha 3$ (IV) NCl et serait impliquée dans sa phosphorylation, événement potentiellement perturbateur de la structure de la chaîne $\alpha 3$ pouvant induire la formation d'anticorps [47]. Plus récemment, il a été montré que la surexpression ciblée de STARDll chez la souris se traduisait par une désorganisation de la membrane basale des glomérules et par le dépôt d'immunoglobulines de type $A$, signe clinique de lupus néphrétique [49]. Très récemment, le rôle potentiel de STARDll dans la résistance médicamenteuse, un problème majeur des traitements chimiques anticancéreux, a été décrit [50]. La protéine STARD1l a été impliquée dans les mécanismes de sensibilité aux molécules utilisées en chimiothérapie, et en particulier au paclitaxel, une molécule de la famille des taxanes qui inhibe la dépolymérisation des microtubules. En effet la diminution de la quantité de STARDll sensibilise les cellules tumorales au paclitaxel, et inversement, sa surexpression induit une résistance à cette drogue. En accord avec un tel rôle de STARD1l, le niveau d'expression de STARD1l est plus élevé dans des tumeurs ovariennes résistantes au traitement. Dans le mécanisme évoqué, STARDll, en modifiant la teneur en céramides des cellules cancéreuses, pourrait moduler leur réponse aux signaux apoptotiques induits par les médicaments. Dans ce contexte, STARD1l pourrait être une cible thérapeutique additionnelle dans les cas de tumeurs résistances aux chimiothérapies.

\section{Conclusion/perspectives}

Le domaine START joue le rôle d'un écran protégeant un lipide hydrophobe d'un environnement hydrophile. II fonctionne comme domaine d'échange de lipides et/ou comme un domaine senseur de lipides. Les protéines à domaine START sont impliquées dans différents mécanismes biologiques : les échanges de lipides entre les compartiments cellulaires, le métabolisme lipidique en particulier pour les protéines qui ont une activité catalytique de type thioestérase associée au domaine START, et dans la transduction des signaux émanant des protéines Rho pour la famille RhoGAP-START. Chez les mammifères, la mouche et le ver, le domaine START occupe invariablement l'extrémité carboxy-terminale, laissant la dernière hélice $\alpha$ libre. Ceci est cohérent avec le modèle d'action proposé. La dernière hélice, en formant un couvercle au-dessus d'une poche profonde où se place le lipide, permettrait l'entrée et la sortie du ligand. Des changements de conformations régis par des contacts avec la membrane, des interactions protéine-protéine ou des phosphorylations/déphosphorylations, permettent de moduler l'activité de transfert des protéines START.

Il est regrettable que les protéines START ne soient pas plus étudiées notamment chez les plantes. En effet, ce domaine est bien plus répandu chez les plantes, organismes qui possèdent un répertoire varié de stérols et par conséquent en ont potentiellement diversifié l'usage. De même, chez les mammifères, certaines protéines START comme STARDI sont extrêmement bien étudiées, alors que d'autres, comme STARD7 ou STARD14/D15, restent très mal caractérisées. Pour toutes ces protéines moins connues, l'identification de leur ligand, de leur patron d'expression et de leur localisation subcellulaire serait importante pour aider à la compréhension de leurs fonctions. Curieusement, chez l'homme, les protéines à domaine START sont fréquemment impliquées dans des pathologies. Hormis le rôle des mutations invalidantes de STARDI dans le syndrome CAH, la protéine STARDIl pourrait être impliquée dans la pathogénie auto-immune et dans la résistance médicamenteuse aux agents anticancéreux. La surexpression ou la perte d'expression de protéines START, relativement fréquente dans les cancers et signe de leur rôle possible dans la promotion et/ou la suppression des cancers, invite à explorer leur fonction de façon plus approfondie. $\diamond$

\section{SUMMARY}

START domain-containing proteins:

a review of their role in lipid transport and exchange

Fifteen START domain-containing proteins exist in mammals. On the basis of their structural homology, this family is divided into several sub-families consisting mainly of non-vesicular intracellular lipid carriers. With the exception of the Thioesterase-START subfamily, the other subfamilies are represented among invertebrates. The START domain is always located in the C-terminus of the protein. It is a module of about 210 residues that binds lipids, including sterols. Cholesterol, 25-hydroxycholesterol, phosphatidylcholine, phosphatidylethanolamine and ceramides are ligands for STARDI/STARD3-6, STARD5, STARD2/STARD10, STARD10 and STARD11, respectively. The lipids or sterols bound by the remaining 7 START proteins are unknown. The START domain can be regarded as a lipid-exchange and/or a lipid-sensing domain. The START domain consists in a deep lipid-binding pocket - that shields the hydrophic ligand from the external aqueous environment - covered by a lid formed by a C-terminal alpha helix. Within the same subgroup, such as the sterols-carriers subgroup, different START domains have similar biochemical properties; however, their expression profile and their subcellular localization distinguish them and are critical for their different biological functions. START proteins act in a variety of distinct physiological processes, such as lipid transfer between intracellular compartments, lipid metabolism and modulation of signaling events. Mutation or misexpression of START proteins is linked to pathological processes, including genetic disorders, autoimmune diseases and cancers. $\diamond$

\section{REMERCIEMENTS}

Nous tenons à nous excuser pour les travaux qui n'ont pu être cités par souci de concision. Nous remercions les membres de l'équipe de Biologie Moléculaire des Cancers du Sein pour leurs 
commentaires sur le manuscrit. F.L. est financé par une Allocation de Recherche du Ministère de l'Enseignement et de la Recherche. L'équipe de C.T. est en partie soutenue par une subvention de la Ligue Contre le Cancer (Comité départemental du Haut-Rhin).

\section{RÉFÉRENCES}

1. Ikonen $\varepsilon$. Cellular cholesterol trafficking and compartmentalization. Nat Rev Mol Cell Biol $2008 ; 9$ : 125-38.

2. Alpy F, Tomasetto C. Give lipids a START: the StAR-related lipid transfer (START) domain in mammals. J Cell Sci $2005 ; 118$ : 2791-801.

3. Clark BJ, Wells J, King SR, Stocco DM. The purification, cloning, and expression of a novel luteinizing hormone-induced mitochondrial protein in MA-10 mouse Leydig tumor cells. Characterization of the steroidogenic acute regulatory protein (StAR). J Biol Chem $1994 ; 269: 28314-22$.

4. Watari H, Arakane F, Moog-Lutz C, et al. MLN64 contains a domain with homology to the steroidogenic acute regulatory protein (StAR) that stimulates steroidogenesis. Proc Natl Acad Sci USA 1997 ; 94 : 8462-7.

5. Moog-Lutz C, Tomasetto C, Regnier CH, et al. MLN64 exhibits homology with the steroidogenic acute regulatory protein (STAR) and is overexpressed in human breast carcinomas. Int J Cancer 1997 ; 71 : 183-91.

6. Soccio RE, Breslow JL. StAR-related lipid transfer (START) proteins: mediators of intracellular lipid metabolism. J Biol Chem 2003 ; $278: 22183-6$.

7. Alpy F, Latchumanan VK, Kedinger V, et al. Functional characterization of the Mental domain. J Biol Chem $2005 ; 280: 17945-52$.

8. Lin D, Sugawara T, Strauss JF 3rd, et al. Role of steroidogenic acute regulatory protein in adrenal and gonadal steroidogenesis. Science $1995 ; 267: 1828-31$.

9. Bose HS, Whittal RM, Ran Y, et al. StAR-like activity and molten globule behavior of StARD6, a male germ-line protein. Biochemistry 2008; 47: 2277-88.

10. Alpy F, Stoeckel ME, Dierich A, et al. The steroidogenic acute regulatory protein homolog MLN64, a late endosomal cholesterol-binding protein. J Biol Chem $2001 ; 276: 4261-9$.

11. Soccio RE, Adams RM, Romanowski MJ, et al. The cholesterol-regulated StarD4 gene encodes a StAR-related lipid transfer protein with two closely related homologues, StarD5 and StarD6. Proc Natl Acad Sci USA $2002 ; 99: 6943-8$.

12. Gomes C, Oh SD, Kim JW, et al. Expression of the putative sterol binding protein Stard6 gene is male germ cell specific. Biol Reprod 2005 ; $72: 651-8$.

13. Munro S. Cell biology: earthworms and lipid couriers. Nature 2003 ; $426: 775-6$.

14. Olayioye MA, Vehring S, Muller P, et al. StarD10, a START domain protein overexpressed in breast cancer, functions as a phospholipid transfer protein. J Biol Chem $2005 ; 280: 27436-42$.

15. Wirtz KW. Phospholipid transfer proteins. Annu Rev Biochem 1991 ; $60: 73-99$.

16. Hanada K, Kumagai K, Yasuda $S$, et al. Molecular machinery for nonvesicular trafficking of ceramide. Nature $2003 ; 426$ : 803-9.

17. Baez JM, Tabas I, Cohen DE. Decreased lipid efflux and increased susceptibility to cholesterol-induced apoptosis in macrophages lacking phosphatidylcholine transfer protein. Biochem J $2005 ; 388: 57-63$.

18. Baez JM, Barbour SE, Cohen DE. Phosphatidylcholine transfer protein promotes apolipoprotein A-I-mediated lipid efflux in Chinese hamster ovary cells. J Biol Chem $2002 ; 277: 6198-206$.

19. Angeletti $S$, Rena V, Nores $R$, et al. Expression and localization of StarD7 in trophoblast cells. Placenta $2008 ; 29: 396-404$.

20. Van Helvoort A, de Brouwer A, Ottenhoff R, et al. Mice without phosphatidylcholine transfer protein have no defects in the secretion of phosphatidylcholine into bile or into lung airspaces. Proc Natl Acad Sci USA $1999 ; 96$ : 11501-6.

21. Wu MK, Hyogo H, Yadav SK, et al. Impaired response of biliary lipid secretion to a lithogenic diet in phosphatidylcholine transfer proteindeficient mice. J Lipid Res 2005 ; $46: 422-31$.

22. Kumagai $K$, Yasuda $S$, Okemoto $K$, et al. CERT mediates intermembrane transfer of various molecular species of ceramides. J Biol Chem 2005 ; $280: 6488-95$.

23. Primeau M, Lamarche-Vane N. Coup d'œil sur les petites GTPases Rho. Med Sci (Paris) $2008 ; 24: 157-62$.
24. Durkin ME, Avner MR, Huh CG, et al. DLC-1, a Rho GTPase-activating protein with tumor suppressor function, is essential for embryonic development. FEBS Lett $2005 ; 579$ : 1191-6.

25. Schrick K, Nguyen D, Karlowski WM, Mayer KF. Start lipid/sterol-binding domains are amplified in plants and are predominantly associated with homeodomain transcription factors. Genome Biol 2004 ; 5 : R4l.

26. Kudo N, Kumagai K, Tomishige N, et al. Structural basis for specific lipid recognition by Cert responsible for nonvesicular trafficking of ceramide. Proc Natl Acad Sci USA 2008 ; $105: 488-93$.

27. Stocco DM, Wang $X$, Jo Y, Manna PR. Multiple signaling pathways regulating steroidogenesis and steroidogenic acute regulatory protein expression: more complicated than we thought. Mol Endocrinol 2005 ; 19 : 2647-59.

28. Chang IY, Kim JH, Hwang G, et al. Immunohistochemical detection of StarD6 in the rat nervous system. Neuroreport $2007 ; 18: 1615-9$.

29. Alpy F, Boulay A, Moog-Lutz C, et al. Metastatic lymph node 64 (MLN64), a gene overexpressed in breast cancers, is regulated by Sp/KLF transcription factors. Oncogene $2003 ; 22: 3770-80$.

30. Qian X, Li G, Asmussen HK, et al. Oncogenic inhibition by a deleted in liver cancer gene requires cooperation between tensin binding and Rho-specific GTPase-activating protein activities. Proc Natl Acad Sci USA 2007 ; 104 : 9012-7.

31. Yamaga M, Sekimata M, Fujii M, et al. A PLCdeltal-binding protein, p122/RhoGAP, is localized in caveolin-enriched membrane domains and regulates caveolin internalization. Genes Cells $2004 ; 9: 25-37$.

32. Rodriguez-Agudo D, Ren S, Hylemon PB, et al. Localization of StarD 5 cholesterol binding protein. J Lipid Res $2006 ; 47: 1168-75$.

33. Fugmann T, Hausser A, Schoffler P, et al. Regulation of secretory transport by protein kinase D-mediated phosphorylation of the ceramide transfer protein. J Cell Biol $2007 ; 178: 15-22$.

34. Saito S, Matsui H, Kawano M, et al. Protein phosphatase 2C (epsilon) is an endoplasmic reticulum integral membrane protein that dephosphorylates the ceramide transport protein CERT to enhance its association with organelle membranes. J Biol Chem $2008 ; 283: 6584-93$.

35. Bose H, Lingappa VR, Miller WL. Rapid regulation of steroidogenesis by mitochondrial protein import. Nature $2002 ; 417: 87-91$.

36. Baker BY, Yaworsky DC, Miller WL. A pH-dependent molten globule transition is required for activity of the steroidogenic acute regulatory protein, StAR. J Biol Chem 2005; $280: 41753-60$.

37. Stocco DM. Clinical disorders associated with abnormal cholesterol transport: mutations in the steroidogenic acute regulatory protein. Mol Cell Endocrinol $2002 ; 191$ : 19-25.

38. Olayioye MA, Hoffmann P, Pomorski T, et al. The phosphoprotein StarD10 is overexpressed in breast cancer and cooperates with $\varepsilon r b B$ receptors in cellular transformation. Cancer Res $2004 ; 64: 3538-44$.

39. Kawai K, Kiyota M, Seike J, et al. START-GAP3/DLC3 is a GAP for RhoA and Cdc42 and is localized in focal adhesions regulating cell morphology. Biochem Biophys Res Commun 2007 ; $364: 783-9$.

40. Wong CM, Lee JM, Ching YP, et al. Genetic and epigenetic alterations of DLC-1 gene in hepatocellular carcinoma. Cancer Res $2003 ; 63: 7646-51$.

41. Yuan BZ, Zhou X, Durkin ME, et al. DLC-1 gene inhibits human breast cancer cell growth and in vivo tumorigenicity. Oncogene $2003 ; 22: 445-50$.

42. Plaumann M, Seitz S, Frege R, et al. Analysis of DLC-1 expression in human breast cancer. J Cancer Res Clin Oncol 2003; 129 : 349-54.

43. Ching YP, Wong CM, Chan SF, et al. Deleted in liver cancer (DLC) 2 encodes a RhoGAP protein with growth suppressor function and is underexpressed in hepatocellular carcinoma. J Biol Chem $2003 ; 278: 10824-30$.

44. $\mathrm{Ng} 10$, Liang ZD, Cao L, Lee TK. DLC-1 is deleted in primary hepatocellular carcinoma and exerts inhibitory effects on the proliferation of hepatoma cell lines with deleted DLC-1. Cancer Res $2000 ; 60: 6581-4$.

45. Yuan BZ, Jefferson AM, Baldwin KT, et al. DLC-1 operates as a tumor suppressor gene in human non-small cell lung carcinomas. Oncogene $2004 ; 23: 1405-11$.

46. Zhou X, Thorgeirsson SS, Popescu NC. Restoration of DLC-1 gene expression induces apoptosis and inhibits both cell growth and tumorigenicity in human hepatocellular carcinoma cells. Oncogene $2004 ; 23: 1308-13$.

47. Raya A, Revert F, Navarro S, Saus J. Characterization of a novel type of serine/threonine kinase that specifically phosphorylates the human goodpasture antigen. J Biol Chem 1999 ; $274: 12642-9$.

48. Hudson BG, Tryggvason K, Sundaramoorthy M, Neilson EG. Alport's syndrome, Goodpasture's syndrome, and type IV collagen. $N$ Engl J Med 2003 ; 348 : 2543-56.

49. Revert F, Merino R, Monteagudo C, et al. Increased Goodpasture antigen-binding protein expression induces type IV collagen disorganization and deposit of immunoglobulin $\mathrm{A}$ in glomerular basement membrane. Am J Pathol 2007 ; 171 : 1419-30.

50. Swanton C, Marani M, Pardo 0 , et al. Regulators of mitotic arrest and ceramide metabolism are determinants of sensitivity to paclitaxel and other chemotherapeutic drugs. Cancer Cell 2007 ; $11: 498-512$

TIRÉS À PART

C. Tomasetto 